\title{
What "Works" in Working Memory? Separate Systems for Selection and Updating of Critical Information
}

\author{
Christoph Bledowski, ${ }^{1,2 *}$ Benjamin Rahm, ${ }^{1 *}$ and James B. Rowe ${ }^{2,3}$ \\ ${ }^{1}$ Institute of Medical Psychology, Goethe University, 60528 Frankfurt am Main, Germany, ${ }^{2}$ Medical Research Council Cognition and Brain Sciences Unit, \\ Cambridge CB2 7EF, United Kingdom, and ${ }^{3}$ Department of Clinical Neurosciences, Cambridge University, Cambridge CB2 2QQ, United Kingdom
}

Cognition depends critically on working memory, the active representation of a limited number of items over short periods of time. In addition to the maintenance of information during the course of cognitive processing, many tasks require that some of the items in working memory become transiently more important than others. Based on cognitive models of working memory, we hypothesized two complementary essential cognitive operations to achieve this: a selection operation that retrieves the most relevant item, and an updating operation that changes the focus of attention onto it. Using functional magnetic resonance imaging, high-resolution oculometry, and behavioral analysis, we demonstrate that these two operations are functionally and neuroanatomically dissociated. Updating the attentional focus elicited transient activation in the caudal superior frontal sulcus and posterior parietal cortex. In contrast, increasing demands on selection selectively modulated activation in rostral superior frontal sulcus and posterior cingulate/precuneus. We conclude that prioritizing one memory item over others invokes independent mechanisms of mnemonic retrieval and attentional focusing, each with its distinct neuroanatomical basis within frontal and parietal regions. These support the developing understanding of working memory as emerging from the interaction between memory and attentional systems.

\section{Introduction}

Working memory is critical for many cognitive tasks and relies on the ability to maintain stable active representations. However, one must also retain the flexibility to highlight momentarily relevant items, enter new items, or discard those no longer needed. This is especially important for the executive functions in working memory such as monitoring or manipulation (Owen et al., 1998; Postle et al., 1999; Smith and Jonides, 1999). How does the brain control the contents of working memory and which operations are involved? One essential operation is to selectively replace some of the contents of working memory with newly acquired information and discard those no longer relevant (Postle et al., 2001; Collette et al., 2006; Roth et al., 2006; Leung et al., 2007; Roth and Courtney, 2007). Other key operations leave the contents of working memory untouched, but require the mental selection or refreshing of one item. These functions award an item in working memory a higher priority for future perception, cognition, or action (Rowe et al., 2000; Nobre et al., 2004; Johnson et al., 2005, 2007; Lepsien et al., 2005; Soto et al., 2007).

\footnotetext{
Received June 2, 2009; revised Aug. 31, 2009; accepted 0ct. 1, 2009.

This study was supported by a research fellowship grant of the Deutsche Forschungsgemeinschaft (DFG BL 931/2-1 to C.B.), the Frankfurt Medical Faculty Intramural Young Investigator Programme (C.B.), the Wellcome Trust (077029 to J.B.R.), and the Medical Research Council Cognition and Brain Sciences Unit. We thank Benjamin Peters for assistance with experiment programming and pilot behavioral measurements and Jochen Kaiser for constant support and advice. We gratefully thank Lucia Melloni from the Max Planck Institute for Brain Research in Frankfurt for the possibility to use the eye tracking system.

${ }^{*}$ C.B. and B.R. contributed equally to this work.

Correspondence should be addressed to Christoph Bledowski, Institute of Medical Psychology, Goethe University, Heinrich-Hoffmann-Strasse 10,60528 Frankfurt am Main, Germany. E-mail: bledowski@em.uni-frankfurt.de. DOI:10.1523/JNEUROSCI.2547-09.2009

Copyright $\odot 2009$ Society for Neuroscience $\quad$ 0270-6474/09/2913735-07\$15.00/0
}

Many studies suggest a close association between working memory and attention, including shared capacity limitations (Marois and Ivanoff, 2005) and common neural organization (Awh and Jonides, 2001; Curtis and D'Esposito, 2003; Courtney, 2004; Postle, 2006; Jonides et al., 2008). Indeed, working memory can be regarded as emerging from the functional interaction of attentional and memory systems. From a cognitive perspective, Cowan (1988) has proposed that the critical process in working memory is attentional and the contents of working memory are conceived as temporarily activated portions of long-term memory. This model has been extended by demonstrating that of all items in working memory, one representation may enter a privileged state of activation termed the focus of attention (Garavan, 1998; McElree, 2001; Oberauer, 2002; Oberauer and Bialkova, 2009). Only this item is readily available for cognitive action, whereas the others require active retrieval first to become able to enter the focus of attention. Hence, in situations that demand switching between different items held in working memory according to their momentary relevance, two control operations will work hand in hand: a selection mechanism that retrieves the relevant item from working memory and an update of the focus of attention with this retrieved item.

Even though these operations of selecting items within working memory and updating of the attentional focus are fundamental to our understanding of the organization of working memory, it is not known whether they have distinct or overlapping neural mechanisms. Often they have been confounded within experimental paradigms. We sought to address this issue by implementing a novel spatial working memory task that incorporated independent manipulations of both selection between items in working memory that calls for their active retrieval and the up- 
dating of the current focus of attention. Our aim was to determine whether selection and updating were manifestations of a single underlying mechanism, with a specific neuroanatomical basis, or whether they reflected independent mechanisms.

\section{Materials and Methods}

Participants. Nineteen right-handed subjects were recruited to the fMRI and behavioral control experiments with normal visual acuity. Subjects gave informed written consent. The study was approved by the Local Research Ethics Committee. fMRI data of two subjects were discarded (head displacement $>10 \mathrm{~mm}$ and abnormalities in the structural scan, respectively). The presented data are from the remaining 17 subjects (nine females, aged 18-35 years). Behavioral data from the fMRI experiment are from 17 subjects; however, data from one subject was incomplete (one session instead of two) due to technical problems. Ten different subjects (four females, age 19-27 years) performed an eye-movement control study.

Study design. The study used a modified delayed-match-to-sample task, consisting of encoding, delay and recognition, with the working memory operations of selection and updating embedded in the delay phase (Fig. 1). At the beginning of each trial, participants saw four black dots (solid squares) presented simultaneously for $3.6 \mathrm{~s}$ on a gray square subtending $3.9^{\circ} \times 3.9^{\circ}$ of visual angle. One dot was presented in each quadrant, at pseudorandom positions. There followed a delay phase during which the participants remembered the exact location of the dots (in Fig. 1 indicated by white squares for illustrative purposes; these were not actually presented to participants). During the delay, a black line serving as a cue appeared for $1.2 \mathrm{~s}$ running across the screen in the vicinity of one or two of the dots. Participants were instructed to focus in mind the position of the dot that was closest to the cue line and maintain it as a target item for a subsequent match-to sample test. This test followed unpredictably after a sequence of two up to five cue lines. The onsets of successive cue lines were separated by an interval of two, three, or four times the repetition time (TR) (1.2 s).

The cue line could run through the vicinity of only one or of two of the remembered dot locations, the latter increasing selection demands on which position was closest. In the course of the series of cue presentations, the same target dot may be cued repeatedly in direct succession, but by different cues. As the target remained unchanged, this would not require updating of the dot position to be kept at focus. Alternatively, a different target location could be indicated, requiring subjects to update the current focus of attention. The independent manipulation of selection and updating demands during the delay phase enabled a repeatedmeasures $2 \times 2$ factorial design with the conditions: high selection/ update $(+\mathrm{S}+\mathrm{U})$, high selection/no update $(+\mathrm{S}-\mathrm{U})$, low selection/ update $(-\mathrm{S}+\mathrm{U})$, and low selection/no update $(-\mathrm{S}-\mathrm{U})$.

In the recognition phase, a single dot was presented for $0.9 \mathrm{~s}$, which with $50 \%$ probability matched the position of the location that the subjects should have as the focus of attention, based on the most recent cue line. Nonmatching probes presented the position of one of the remaining sample dots. Subjects judged whether the probe matched the most recently cued position and responded "yes/no" as quickly as possible by button press with their right index or right ring finger. Fast responses were induced by imposing a response deadline of $0.9 \mathrm{~s}$. After the probe presentation, participants received a feedback for $0.6 \mathrm{~s}$ whether they had responded correctly (green square) or not (red square). Both late and incorrect responses were fed back as errors. The unpredictable number of cue presentations during the delay phase (two up to five) and the emphasis on quick responses served to ensure that each single-cue presentation would be attended to and that the required memory operations were performed. Each trial ended with a rest period varying randomly among 2.1, 3.3, and $4.5 \mathrm{~s}$. Subjects were instructed to maintain fixation to the center of the gray background square throughout (although there was no fixation cue).

As the need for updating was determined by the immediately preceding cue, the first cue presentation of each trial was of no interest. In consequence, a trial included the remaining one to four events of interests. A total of 96 trials were presented, resulting in an average of 60 repetitions for each of the four experimental conditions. The order of cues was pseudorandomized, such that each cue type was followed with equal probability by each of the four cue types.

Presentation software (version 11.3) was used for stimulus presentation, response recording, and scanner and eye tracker synchronization. In fMRI, visual stimuli were projected onto a screen using a Christie video projector LX35 with custom lens at resolution $1024 \times 768$ backprojected onto a mirror mounted on the head coil.

$f M R I$ acquisition and analysis. Functional and anatomical images were acquired at the MRC Cognition and Brain Sciences Unit on a Siemens 3 T TIM Trio scanner. Functional images were collected in two sessions of 1070 volumes each, using a blood oxygen level-dependent (BOLD)sensitive $\mathrm{T} 2 *$-weighted echo-planar sequence [time echo $(\mathrm{TE})=25 \mathrm{~ms}$; flip angle $(\mathrm{FA})=78^{\circ}$; matrix $\left.=64 \times 64\right]$ with twenty-one 5 -mm-thick $(3 \times 3 \mathrm{~mm}$ in-plane, $0.5 \mathrm{~mm}$ skip) slices and a TR of $1.2 \mathrm{~s}$. A highresolution, magnetization-prepared rapid-acquisition gradient echo $\left(\mathrm{TR}=2250 \mathrm{~ms}, \mathrm{TE}=2.99 \mathrm{~ms}, \mathrm{FA}=9^{\circ}\right.$, inversion time $900 \mathrm{~ms}, 256 \times$ $256 \times 192$ isotropic $1 \mathrm{~mm}$ voxels) and a single volume turbo spine echo $(\mathrm{TR}=5060 \mathrm{~ms}, \mathrm{TE}=102 \mathrm{~ms}, \mathrm{FA}=140,28 \times 4 \mathrm{~mm}$ slices $)$ structural image were obtained for purpose of coregistration, normalization, and assurance of structural normality after the first functional session. 
FMRI data were analyzed using Statistical Parametric Mapping (SPM5; Wellcome Department of Cognitive Neurology). Preprocessing included slice-time correction, realignment to the first image, nonlinear normalization to the Montreal Neurological Institute (MNI) template, resampling of voxel size to $3 \times 3 \times 3 \mathrm{~mm}$, and spatial smoothing with an $8 \mathrm{~mm}$ full-width at half-maximum Gaussian kernel.

For the design matrix, time points were defined to represent different task phases of a single trial (encoding, first cue presentation, second to fifth cue presentations, during the memory delay and a probe). At the first level, each of these task phases was modeled using a finite impulse response approach. We used 12 time points with an interval of $1.2 \mathrm{~s}$ (corresponding to the TR). The first cue always demanded updating, because no other cue had preceded it so that the focus of attention had to be shifted from central fixation to one of the remembered locations. To avoid biases, the first cues of each trial were modeled as a separate effect of no interest. All subsequent cue presentations in series (second, third, fourth, and fifth, depending on the total number of cues in a specific trial) were defined as cue of interests and represented the independent combinations of selection and updating demands in a pseudorandom order. They were modeled by separate regressor for each condition. The probe presentation was modeled separately for "match" and "nonmatch" trials. In addition, a block of variable duration was used to model the encoding/ maintenance period, which spanned the whole time window from the onset of the to-be-remembered positions to the onset of the probe stimulus (including persistent activation). Because there were between two and five cue presentations per trial, the duration of the block varied across trials. The hemodynamic response of this block was also segmented into 12 bins (due to its extended duration, one bin did not correspond to one scan for this part of the task).

For each cue of interest $(+S+U,+S-U,-S+U$, and $-S-U)$, individual contrasts versus implicit baseline for scans $4-6$ after cue onset were computed. This corresponds to a time window of 3.6-7.2 $\mathrm{s}$ after cue that covers the peak of the hemodynamic response even in the presence of some interregional variability.

The resulting parameter maps from each subject were entered into a second-level repeated-measurements ANOVA analysis with subjects as a random factor and the within-subjects factors of scan (fourth through sixth scan), selection (high vs low), and updating (updating, no updating). Using $F$ contrasts, brain regions responsive to changes in selection (high vs low selection) and updating (vs no updating) demands were identified by a significant main effect of selection and updating present at the fourth, fifth, or sixth scan. The statistical $\operatorname{SPM}(F)$ maps were thresholded at $p<0.05$ [false discovery rate (FDR) corrected; cluster threshold $=10$ voxels $]$ and visualized on a surface reconstruction of the SPM canonical single-subject brain using the BrainVoyager QX software (Brain Innovation).

In addition, we used a conjunction analysis to identify regions with significant activation for both selection and updating as well as to identify brain areas significantly activated by every single-cue condition (see supplemental Fig. 1, available at www.jneurosci.org as supplemental material). Conversely, we used an exclusive masking approach to identify regions with a significant effect of selection ( $p<0.05$ corrected) but no more than a slight effect of updating (no more than a trend at $p<0.1$ uncorrected).

Region of interest (ROI) analyses were conducted to compare the effect sizes revealed by voxel-based statistics. Spherical ROIs (radius 6 $\mathrm{mm}$ ) were centered on peak coordinates of clusters activated by the main effect of selection and updating ( $p<0.05$; FDR) (Table 1). MarsBar toolbox (http://marsbar.sourceforge.net) was used to extract for each condition the first eigenvariate of the BOLD signal and to estimate the individual effect size for the average of scans 4,5 , and 6 after cue onset. Effect sizes for selection and updating were then compared using a post hoc $t$ test.

Control studies. Before fMRI scanning each subject underwent a behavioral measurement. The stimuli and task were the same as in the fMRI experiment, except for the following differences. In addition to the responses to the probe stimulus subjects were asked to confirm via right middle finger button press when they had selected in mind the dot closest to the cue line after each single-cue presentation. This time was taken as
Table 1. List of cortical regions for selection and updating

\begin{tabular}{cllc}
\hline Brain region & Side & Z score & MNI coordinates $(x, y, z$ in $\mathrm{mm})$ \\
\hline Selection & & & \\
CSFS & Left & 3.80 & $-21,-6,51$ \\
& Right & 4.28 & $24,-3,51$ \\
PPC & Left & 4.48 & $-18,-66,57$ \\
& Right & 5.21 & $18,-60,57$ \\
rSFS & Right & 3.57 & $42,-36,42$ \\
PCC & Left & 4.02 & $-24,30,36$ \\
PCN & Left & 4.46 & $-21,-30,39$ \\
& Left & 4.58 & $-9,-48,36$ \\
vmPFC & Right & 4.13 & $6,-45,33$ \\
& Left & 3.82 & $-3,54,-6$ \\
IPL & Right & 3.69 & $18,57,24$ \\
PHG & Right & 4.12 & $18,51,-3$ \\
Updating & Left & 4.15 & $-51,-66,36$ \\
CSFS & Left & 4.65 & $-27,0,-21$ \\
& & & \\
PPC & Left & 5.12 & $-24,-3,57$ \\
& Right & 3.88 & $27,-3,54$ \\
& Left & 5.26 & $-18,-66,54$ \\
vmPFC & Right & 5.45 & $18,-60,57$ \\
BG & Left & 4.00 & $-33,-39,42$ \\
Selection $\times$ updating & Right & 3.74 & $42,-39,54$ \\
n.S. & Right & 3.84 & $27,63,12$ \\
& Left & 4.07 & $-21,12,-6$ \\
& & & \\
\hline & & & \\
& & &
\end{tabular}

fMRI results of SPM $(F)$ contrasts ( $p<0.05$, FDR corrected) for the effects of modulation of selection (high selection vs low selection) and updating (updating vs no updating) at the time of cue presentations within the memory delay. The independent experimental manipulations of selection and updating were associated with differential activations in separate sets of frontoparietal cortical regions. There was no significant interaction between the factors of selection and updating. vmPFC, Ventromedial prefrontal cortex; IPL, inferior parietal lobule; PHG, parahippocampal gyrus; BG, basal ganglia/putamen.

a behavioral index of the time needed for the demanded operations and was used as a dependent variable in a 2 (high, low selection) $\times 2$ (updating, no updating) repeated-measures ANOVA. The intertrial interval was fixed at $4.5 \mathrm{~s}$. In total, 36 trials were presented, which resulted in an average of 22.5 repetitions of each cue type.

Ten different subjects performed an eye-movement control study, which consisted of a working memory and a baseline fixation session. In the working memory session, subjects performed the very working memory task that was used in fMRI (but only 48 instead of 96 trials, resulting in an average of $\sim 30$ repetitions of each of the four experimental conditions). As in the fMRI study, subjects were instructed to permanently maintain their fixation on the center of the gray display box. In the baseline fixation session, 12 trials of the working memory task were presented with identical stimulus and timing parameters, but subjects were only asked to maintain fixation to the center of the gray display box and did not perform any task. This allowed for an assessment of spontaneous saccade rates as a baseline measure in the absence of all working memory demands. Due to technical problems, baseline fixation data of one subject is missing.

Recording of eye movements was conducted binocularly at $1000 \mathrm{~Hz}$ using the video-based infrared EyeLink 1000/2K Desktop system (SR Research). Nine-point calibration was performed at the beginning of each session. The head position was maintained using a chin rest. During the cue presentation $(1.2 \mathrm{~s})$, saccades were detected by the EyeLink software, using eye-movement thresholds of $22 \%$ for velocity and $4000 \% \mathrm{~s}^{2}$ for acceleration. For statistical analysis, we calculated the average saccade rate per cue presentation separately for each subject and experimental condition and performed two-way repeated-measures ANOVAs with selection and updating as within-group factors. For the comparison of eye movements between the working memory and the baseline fixation session, we used a $t$ test for paired samples on the saccade rate averaged across all cue presentations in each session separately. 


\section{Results}

Behavioral results

To obtain a direct behavioral measure of the cue task performance, subjects underwent separate behavioral testing before fMRI scanning. The stimuli and task were the same as during scanning, except that at every presentation of a cue, subjects additionally indicated by key press the time point when they had finished selection/ updating. This time was used as a dependent variable and analyzed with a 2 (high, low selection) $\times 2$ (updating, no updating) repeated-measures ANOVA. The results are shown in Figure 2. As expected, subjects were slower after high selection cues $(844.2 \pm 42.9 \mathrm{~ms}$, mean $\pm \mathrm{SEM}$, for $+\mathrm{S}+\mathrm{U}$ and $787.8 \pm 46.0 \mathrm{~ms}$ for $+\mathrm{S}-\mathrm{U}$ vs $770.1 \pm 39.9 \mathrm{~ms}$ for $-\mathrm{S}+\mathrm{U}$ and $702.7 \pm 35.2 \mathrm{~ms}$ for $-\mathrm{S}-\mathrm{U}$; main effect of selection: $\left.F_{(1,16)}=25.3, p=0.000\right)$. Also, they were slower when updating the cued location (main effect of updating: $\left.F_{(1,16)}=13.9, p=0.002\right)$. Importantly, we did not observe any interaction between selection and updating $\left(F_{(1,16)}=0.3, p=\right.$ 0.602 ), i.e., subjects were slower when they updated the focus of attention in working memory than when no update was needed independent of selection demands and vice versa. These results clearly indicate that demands on selection and updating were successfully manipulated and independent.

In fMRI we sought to avoid extensive motor-related activations during cue-induced memory operations. We therefore required overt responses only to the probe item at the end of each trial (recognition phase). Behavioral data from the fMRI study showed that recognition accuracy was high $(85.6 \pm 1.6 \%)$ with reaction times (RTs) lying clearly below the response deadline $(618.2 \pm 13.1 \mathrm{~ms})$. Importantly, RTs were faster to probes that matched the current focus of attention than to nonmatching positions $(596.8 \pm 14.6 \mathrm{~ms}$ vs $639.6 \pm 15.4 \mathrm{~ms}$; paired $t$ test, $t=$ $-2.9 ; p=0.010)$. Thus, as commonly observed in attentional cueing paradigms (Posner, 1980), devoting spatial attention to a position accelerates detection of stimuli presented there. In consequence, these results indicate that participants performed the intended selecting or updating at every cue presentation and maintained the selected item as a target for recognition.

\section{fMRI results}

To characterize the neural correlates of working memory selection and updating, we analyzed the $\mathrm{FMRI} B \mathrm{BLD}$ responses to the four cue types $(+S+U,+S-U,-S+U$, and $-S-U)$. In a first step, to identify regions responsive to demands of either selection or updating, we computed the respective main effects $(\operatorname{SPM}(F)$ map, $p<0.05$, FDR corrected; cluster threshold $=10$ voxels), identifying activation differences in scans 4, 5, or 6 after cue onset ( TR $=1.2 \mathrm{~s})$ covering the peak of the hemodynamic response.

In accordance with our hypothesis, selection between items in working memory and the updating of the currently focused target item were associated with differential activity in distinct brain regions. The brain areas sensitive to the manipulation of selection regardless of updating comprised left and right caudal superior frontal sulcus (cSFS), posterior parietal cortex (PPC) including superior parietal lobe and inferior parietal sulcus, left rostral superior frontal sulcus (rSFS), posterior cingulate cortex (PCC), precuneus $(\mathrm{PCN})$, ventromedial prefrontal frontal cortex, and left inferior parietal lobe (Fig. 3). b

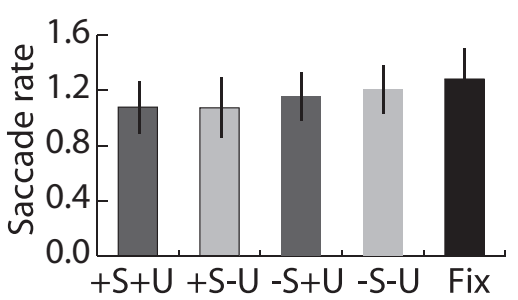

In contrast, whenever an update of the focus of attention was needed, regardless of the selection demands, we observed differential activation of the bilateral cSFS and PPC. For a complete list of active areas, see Table 1. In addition, no brain area exhibited a significant interaction between effects of selection and updating at standard threshold $[\operatorname{SPM}(F)$ map, $p<0.05$, FDR corrected; cluster threshold $=10$ voxels] or a more liberal voxelwise threshold ( $p<0.001$, uncorrected).

All four cue types invoked some common processes, similar to those identified by Rowe et al. (2000) in response to the cue line. These common elements were identified by a conjunction analysis across all four cues $(+\mathrm{S}+\mathrm{U}$ and $+\mathrm{S}-\mathrm{U}$ and $-\mathrm{S}+\mathrm{U}$ and $-\mathrm{S}-\mathrm{U}$, each vs baseline, FDR $p<0.05)$ and included activation of the middle frontal gyrus of dorsolateral prefrontal cortex (dlPFC) but not rSFS (see supplemental Fig. 1, available at www. jneurosci.org as supplemental material).

The previous analyses of thresholded responses do not yet allow for conclusions concerning functional selectivity, i.e., whether a region is significantly more responsive to demands on selection versus updating. We therefore directly compared the effect sizes of selection versus updating. Formally, this was done by extracting the individual effect sizes ( $\beta$ values) from regions of interest (radius $6 \mathrm{~mm}$ ) centered on the local maxima of the main effects of the previous analysis and entering these in dependent samples $t$ tests. These revealed that BOLD signal changes in left rSFS and PCC/PCN were significantly more pronounced in selection than in updating (for rSFS: $t=2.73, p=0.010$; for PCC/ PCN: $t=2.92, p=0.015$ ) (see also Fig. 3). There was no region that was significantly more active during updating than selection.

Updating-related activity in these regions may however still be present, albeit subthreshold and less strong than for selection. Thus, to test the extent at which they were strictly exclusive for selection, we used an exclusive masking approach. We examined which of the selection-related activations do not exhibit an effect of updating even at a very liberal threshold of $p<0.1$ uncorrected. Clusters in left rSFS and PCC/PCN previously shown to be selectively enhanced for selection were unaltered. Hence, we infer that these areas are activated exclusively in response to selection demands.

To summarize, we observed two distinct activation patterns subserving updating and selection processes, respectively. A common set of regions including bilateral cSFS and PPC showed stronger activation in response to both selection and updating (this result was also supported by a conjunction analysis) (supplemental Table 1, available at www.jneurosci.org as supplemen- 
a
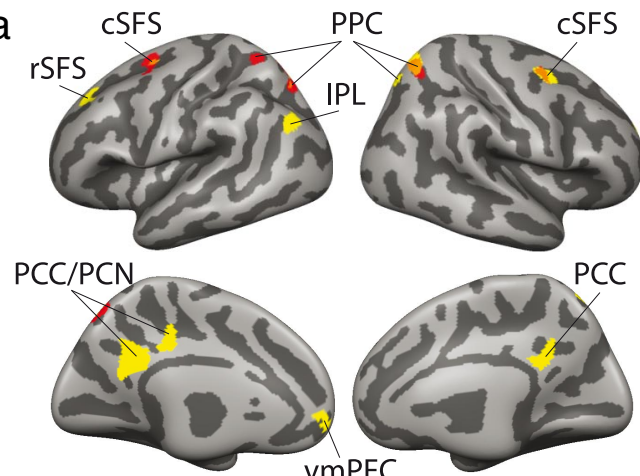

PCC
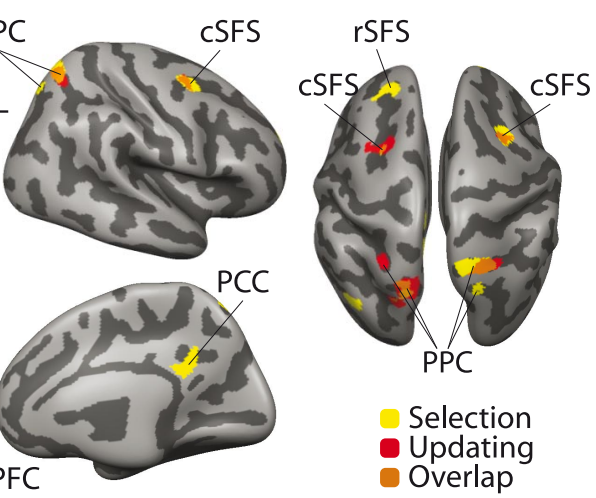

$\mathrm{b}$
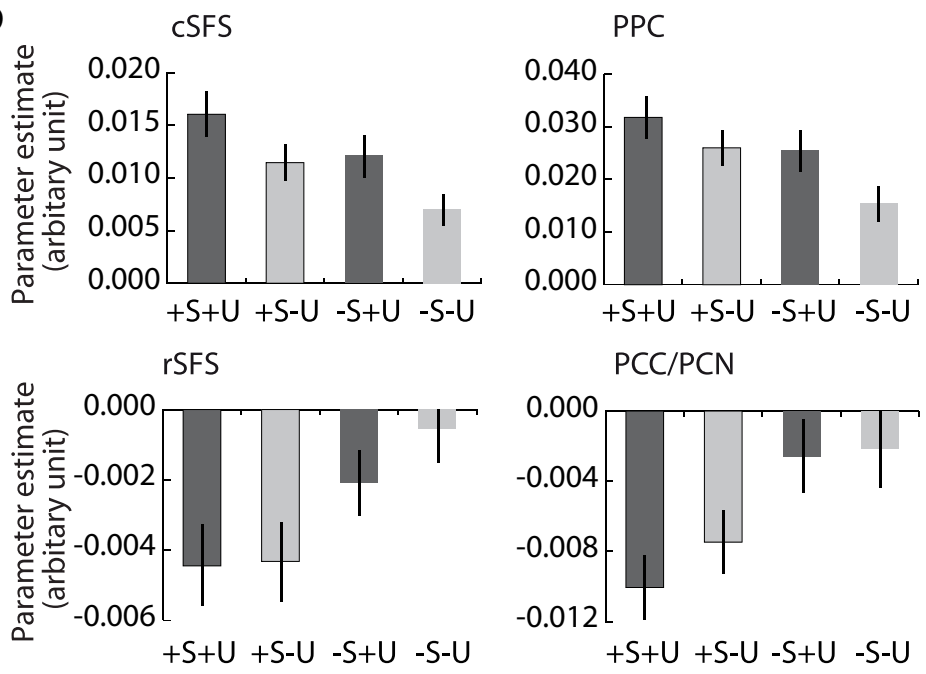

Figure 3. Dissociable neuronal correlates of selection and updating. $\boldsymbol{a}$, Modulation of regional activation by selection (yellow) and updating (red) projected onto the surface cortex reconstruction of the SPM canonical single-subject brain. Areas of overlap are shown in orange. The full set of coordinates is listed in Table 1. Note that no brain region was sensitive to an interaction between updating and selection. $\boldsymbol{b}$, Estimated effect size coefficients (mean \pm SEM) for the regions of interest showing either main effects of selection and updating (CSFS and PPC) or an exclusive response to selection modulation only (rSFS and PPC/PCN).

tal material), but did not show an interaction between them. In contrast, activations in left rSFS and PCC/PCN were exclusively sensitive to demands on selection between items held in working memory.

\section{Eye-movement control study}

Bilateral cSFS and PPC were significantly activated during updating as well as selection, attributable to shifts of attention. However, activation of these regions is also observed when subjects generate overt eye movements (Corbetta et al., 1998; Konen and Kastner, 2008). Thus, differences between conditions might be due to differences in eye movements. To evaluate this alternative interpretation of our data, we conducted a separate eye-movement control study. For this we recorded eye movements in 10 subjects with a high spatial accuracy and temporal resolution using a video-based infrared eye tracker (for details, see Materials and Methods) and analyzed the number of saccades participants had made. Behavioral data were similar to the fMRI results (recognition accuracy: $87.9 \pm 2.9 \%$, mean \pm SEM; RTs were faster to match than to nonmatch probes: $635.6 \pm 33.6 \mathrm{~ms}$ vs $686.2 \pm 43.1$ ms; paired $t$ test, $t=-3.5$; $p=0.006$ ), indicating that performance of the working memory task was comparable across different groups and experimental settings.

Importantly, the eye-movement data showed that during the $1.2 \mathrm{~s}$ of cue presentation, the average number of saccades did not differ between the experimental conditions (Fig. 2; supplemental Fig. 2, available at www.jneurosci.org as supplemental material). This was confirmed by a repeatedmeasures ANOVA that failed to reveal a main effect of selection $\left(F_{(1,9)}=2.3, p=\right.$ 0.170 ; number of saccades per cue presentation: $1.08 \pm 0.60$, mean \pm SEM, for $+\mathrm{S}+\mathrm{U}$ and $1.07 \pm 0.70$ for $+\mathrm{S}-\mathrm{U}$ vs $1.16 \pm 0.56$ for $-S+U$ and $1.21 \pm 0.57$ for $-\mathrm{S}-\mathrm{U})$ or updating $\left(F_{(1,9)}=0.5, p=\right.$ $0.509)$. Also, we did not observe any interaction between the two factors $\left(F_{(1,9)}=0.4\right.$, $p=0.534)$. Moreover, the overall saccade rate was similar when subjects just passively viewed the task stimulation (not requiring any working memory operations and shifts of spatial attention) $(t=0.9$, $p=0.377$; average saccade rate: $1.21 \pm$ 0.57 for working memory and $1.28 \pm 0.68$ for fixation session). Thus, we conclude that the activity changes observed in cSFS and PPC were modulated by demands on selection and updating and are highly unlikely to have arisen from differential numbers of eye movements.

\section{Discussion}

Working memory supports many higher cognitive functions by keeping a small number of items available in a state of active neural representation, and by selecting and attending preferentially to that item that is momentarily most relevant. This close relationship between memory and attention is reflected in contemporary models of working memory (Cowan, 1988; Awh and Jonides, 2001; Curtis and D’Esposito, 2003; Courtney, 2004; McElree, 2006; Postle, 2006; Jonides et al., 2008). Extending these models, we found dissociable neural mechanisms of selection and updating. These two processes were subserved by different sets of frontoparietal cortical regions with independent behavioral effects, supporting the hypothesis that selection and updating are distinct working memory operations.

In our paradigm, the selection of a new position based on the cue stimuli within each trial led to an updating of the attentional focus (required for speeded recognition). This update, or change in the focus of attention, was associated with activations in cSFS and PPC, both regions associated with spatial attention in tasks without strong memory demands (Kastner and Ungerleider, 2000; Corbetta et al., 2002; Yantis and Serences, 2003). The results of our eye-tracking control experiment strongly suggest that these activations were not due to potential differences in the number of saccades induced by our task manipulations. Rather, the activations support the hypothesis that spatial information is retained by attention-based rehearsal, i.e., by systematic shifts of attention to the to-be-remembered positions (Awh and Jonides, 2001; Corbetta and Shulman, 2002; Postle et al., 2004; Awh et al., 2006; Jonides et al., 2008). In that sense, rehearsal of spatial memoranda could be conceived as a series of updating operations in cSFS and PPC. Since activation in these regions has also been associated with directing attention to nonspatial features of visual 
stimuli and mental representations (Lepsien et al., 2005; Lepsien and Nobre, 2007), their role may not be limited to the spatial domain.

The suggestion that PPC and cSFS are implicated in updating the attentional focus is further supported by fMRI studies on updating the working memory contents with nonspatial information like faces and houses (Roth et al., 2006; Roth and Courtney, 2007). In these studies by Roth and colleagues, updating was defined as the cue-based replacement of a currently memorized item in working memory with a newly acquired input. In contrast, in our study the updating event required a switch of the attention focus between items constantly held in working memory, without the presentation of a new item. This difference may explain why updating of the working memory contents was associated with additional activations of prefrontal (inferior frontal gyrus, pre-supplementary motor area) and extrastriate cortex (inferior occipitotemporal and parahippocampal gyrus). We propose that shifting the attentional focus, subserved by cSFS and PPC, represents updating of attentional focus common in both types of task, while the extrastriate, ventrolateral, and medial frontal cortex mediate visual processing and encoding of new information into working memory. This interpretation is also supported by results of Postle et al. (2001) and Montojo and Courtney (2008). For example, Montojo and Courtney recently showed that again, the updating of numbers and rules (mathematical operations) activated regions associated with the shifts of attentional focus. In addition, the update of a number preferentially activated the PPC (also associated with numerosity) and the update of a rule the inferior frontal gyrus [an area responsive to rule switching (Derrfuss et al., 2005)]. Together, the converging evidence between studies using different materials and operational definitions of updating suggests that cSFS and PPC may form the neuronal basis of a supramodal attentional updating mechanism, whereas other regions show stronger task and material specificity between studies. We conclude that the changes of the attentional focus represent the core mechanism underlying different types of updating, emphasizing the role of attention in the control of working memory.

Activation in cSFS and PPC was not specific for updating, but was additionally modulated by demands on selection between items in working memory. This supports recent cognitive conceptions that to make a memory representation amenable to operation, such as evaluating its distance to a cue line, it has to be put into the focus of attention (Garavan, 1998; McElree, 2001; Oberauer, 2002; Oberauer and Bialkova, 2009). In consequence, with more items to select from, more activation is to be expected in regions subserving the updating of the attentional focus. Thus, while the data alone may argue for a role of cSFS and PPC in both updating and selection, the apparent effects of selection in these regions may be attributable to increased concomitant updating demands under high selection. In line with this interpretation, the conjunction of selection- and updating-related activations was limited to regions previously implicated in attentional processing.

Critically, we found selection-specific modulation of activity in a distinct set of frontoparietal areas. Differential activity in the rSFS and PCC/PCN was not driven by updates of spatial attention. In our paradigm, selection demands manipulated the need for memory retrieval: the cue line ran close to either one or two positions held in working memory. Behavioral research indicates that the access to representations that are outside of the current focus of attention is mediated by the same mechanism that retrieves representation from episodic memory (McElree, 2001,
2006). Using fMRI, Nee and Jonides (2008) showed that selection of verbal items outside of the focus of attention recruits brain regions similar to those implicated in the retrieval from longterm memory, in contrast to an item already at the focus of attention (the most recently presented item). However, in that study, the neural bases of memory retrieval and updating could not be distinguished.

Our high-selection events also involve a decision of which of the two retrieved positions is closer to the cue line. Our interpretation is that the activations in rSFS and PCC/PCN associated exclusively with selection (not updating) comprise both regions that play a role in memory retrieval as well as decision making. There is a high degree of overlap between our selection-sensitive regions, the activation foci from studies on memory retrieval, and areas involved in perceptual decision making. For example, the results of both the meta-analysis of recollection success by Wagner et al. (2005) and Heekeren's studies of perceptual decision making (Heekeren et al., 2004, 2006) reveal common activations of PCC/PCN and rSFS. This may be due to common requirements for accessing internal representations, evidence accumulation, and decision making in all three lines of research using functional neuroimaging. Other methods might allow a classification of these regions as primarily memory or decision related: e.g., lesions in PCN/retrosplenial cortex impair retrieval of information from episodic memory (Valenstein et al., 1987), while single-unit recording from monkey prefrontal cortex demonstrates accumulation of evidence to compute a decision (Kim and Shadlen, 1999). It is possible therefore that in the healthy human brain the PCC/PCN subserves memory retrieval, whereas the left rSFS enables decision about retrieved items.

Previous studies of working memory using similar cues have shown activations of the dlPFC (middle frontal gyrus) with the selection or "refreshing" of a stimulus representation in memory but not the maintenance of stimulus locations or directly specified response selection (Rowe et al., 2000; Rowe and Passingham, 2001; Johnson et al., 2007). In the present study, similar cues appeared in succession two to five times per trial, and processes common to all cue types were identified by the conjunction analysis $(+\mathrm{S}+\mathrm{U}$ and $+\mathrm{S}-\mathrm{U}$ and $-\mathrm{S}+\mathrm{U}$ and $-\mathrm{S}-\mathrm{U}$, each vs baseline) with activation in the same dlPFC region as previous studies (see supplemental Fig. 1, available at www.jneurosci.org as supplemental material). This suggests that the dlPFC activation for selection is present even with relatively low selection demands and does not depend on the number of reactivated or attended items between which one has to select. In contrast, activity of the rSFS was not equivalent across all cue types, but varied with increasing demands on selection between remembered items in memory. Finally we note that a potential confound in previous studies of selection in working memory has been the coincidence between selection events and the transition between phases of a trial. It could have been argued that the previously reported prefrontal activations reflected a switch between cognitive sets linked to stimulus/memory and response phases respectively (Rushworth et al., 2005). However, in our paradigm, the series of selection/updating operations in working memory were not confounded by the transition from memory to response phases, and the presence of mnemonic selection remains the relevant function for activation of dIPFC.

In conclusion, the present study provides behavioral and neurophysiological evidence for a functional and anatomical distinction between selection and updating operations within working memory. Selection and updating both contribute to control of working memory but recruit dissociable neural substrates that 
have previously been related to attention and memory systems. Our results support and extend a generic working memory model based on interactions between distributed attentional and mnemonic systems.

\section{References}

Awh E, Jonides J (2001) Overlapping mechanisms of attention and spatial working memory. Trends Cogn Sci 5:119-126.

Awh E, Vogel EK, Oh SH (2006) Interactions between attention and working memory. Neuroscience 139:201-208.

Collette F, Hogge M, Salmon E, Van der Linden M (2006) Exploration of the neural substrates of executive functioning by functional neuroimaging. Neuroscience 139:209-221.

Corbetta M, Shulman GL (2002) Control of goal-directed and stimulusdriven attention in the brain. Nat Rev Neurosci 3:201-215.

Corbetta M, Akbudak E, Conturo TE, Snyder AZ, Ollinger JM, Drury HA, Linenweber MR, Petersen SE, Raichle ME, Van Essen DC, Shulman GL (1998) A common network of functional areas for attention and eye movements. Neuron 21:761-773.

Corbetta M, Kincade JM, Shulman GL (2002) Neural systems for visual orienting and their relationships to spatial working memory. J Cogn Neurosci 14:508-523.

Courtney SM (2004) Attention and cognitive control as emergent properties of information representation in working memory. Cogn Affect Behav Neurosci 4:501-516.

Cowan N (1988) Evolving conceptions of memory storage, selective attention, and their mutual constraints within the information processing system. Psychol Bull 104:163-191.

Curtis CE, D'Esposito M (2003) Persistent activity in the prefrontal cortex during working memory. Trends Cogn Sci 7:415-423.

Derrfuss J, Brass M, Neumann J, von Cramon DY (2005) Involvement of the inferior frontal junction in cognitive control: meta-analyses of switching and Stroop studies. Hum Brain Mapp 25:22-34.

Garavan H (1998) Serial attention within working memory. Mem Cognit 26:263-276.

Heekeren HR, Marrett S, Bandettini PA, Ungerleider LG (2004) A general mechanism for perceptual decision-making in the human brain. Nature 431:859-862.

Heekeren HR, Marrett S, Ruff DA, Bandettini PA, Ungerleider LG (2006) Involvement of human left dorsolateral prefrontal cortex in perceptual decision making is independent of response modality. Proc Natl Acad Sci U S A 103:10023-10028.

Johnson MK, Raye CL, Mitchell KJ, Greene EJ, Cunningham WA, Sanislow CA (2005) Using fMRI to investigate a component process of reflection: prefrontal correlates of refreshing a just-activated representation. Cogn Affect Behav Neurosci 5:339-361.

Johnson MR, Mitchell KJ, Raye CL, D’Esposito M, Johnson MK (2007) A brief thought can modulate activity in extrastriate visual areas: top-down effects of refreshing just-seen visual stimuli. Neuroimage 37:290-299.

Jonides J, Lewis RL, Nee DE, Lustig CA, Berman MG, Moore KS (2008) The mind and brain of short-term memory. Annu Rev Psychol 59:193-224.

Kastner S, Ungerleider LG (2000) Mechanisms of visual attention in the human cortex. Annu Rev Neurosci 23:315-341.

Kim JN, Shadlen MN (1999) Neural correlates of a decision in the dorsolateral prefrontal cortex of the macaque. Nat Neurosci 2:176-185.

Konen CS, Kastner S (2008) Representation of eye movements and stimulus motion in topographically organized areas of human posterior parietal cortex. J Neurosci 28:8361-8375.

Lepsien J, Nobre AC (2007) Attentional modulation of object representations in working memory. Cereb Cortex 17:2072-2083.

Lepsien J, Griffin IC, Devlin JT, Nobre AC (2005) Directing spatial attention in mental representations: interactions between attentional orienting and working-memory load. Neuroimage 26:733-743.

Leung HC, Oh H, Ferri J, Yi Y (2007) Load response functions in the human spatial working memory circuit during location memory updating. Neuroimage 35:368-377.

Marois R, Ivanoff J (2005) Capacity limits of information processing in the brain. Trends Cogn Sci 9:296-305.

McElree B (2001) Working memory and focal attention. J Exp Psychol Learn Mem Cogn 27:817-835.

McElree B (2006) Accessing recent events. In: The psychology of learning and motivation (Ross BH, ed). San Diego: Academic.

Montojo CA, Courtney SM (2008) Differential neural activation for updating rule versus stimulus information in working memory. Neuron 59:173-182.

Nee DE, Jonides J (2008) Neural correlates of access to short-term memory. Proc Natl Acad Sci U S A 105:14228-14233.

Nobre AC, Coull JT, Maquet P, Frith CD, Vandenberghe R, Mesulam MM (2004) Orienting attention to locations in perceptual versus mental representations. J Cogn Neurosci 16:363-373.

Oberauer K (2002) Access to information in working memory: exploring the focus of attention. J Exp Psychol Learn Mem Cogn 28:411-421.

Oberauer K, Bialkova S (2009) Accessing information in working memory: Can the focus of attention grasp two elements at the same time? J Exp Psychol Gen 138:64-87.

Owen AM, Stern CE, Look RB, Tracey I, Rosen BR, Petrides M (1998) Functional organization of spatial and nonspatial working memory processing within the human lateral frontal cortex. Proc Natl Acad Sci U S A 95:7721-7726.

Posner MI (1980) Orienting of attention. Q J Exp Psychol 32:3-25.

Postle BR (2006) Working memory as an emergent property of the mind and brain. Neuroscience 139:23-38.

Postle BR, Berger JS, D’Esposito M (1999) Functional neuroanatomical double dissociation of mnemonic and executive control processes contributing to working memory performance. Proc Natl Acad Sci U S A 96:12959-12964.

Postle BR, Berger JS, Goldstein JH, Curtis CE, D’Esposito M (2001) Behavioral and neurophysiological correlates of episodic coding, proactive interference, and list length effects in a running span verbal working memory task. Cogn Affect Behav Neurosci 1:10-21.

Postle BR, Awh E, Jonides J, Smith EE, D’Esposito M (2004) The where and how of attention-based rehearsal in spatial working memory. Brain Res Cogn Brain Res 20:194-205.

Roth JK, Courtney SM (2007) Neural system for updating object working memory from different sources: sensory stimuli or long-term memory. Neuroimage 38:617-630.

Roth JK, Serences JT, Courtney SM (2006) Neural system for controlling the contents of object working memory in humans. Cereb Cortex 16:15951603.

Rowe JB, Passingham RE (2001) Working memory for location and time: activity in prefrontal area 46 relates to selection rather than maintenance in memory. Neuroimage 14:77-86.

Rowe JB, Toni I, Josephs O, Frackowiak RS, Passingham RE (2000) The prefrontal cortex: response selection or maintenance within working memory? Science 288:1656-1660.

Rushworth MF, Passingham RE, Nobre AC (2005) Components of attentional set-switching. Exp Psychol 52:83-98.

Smith EE, Jonides J (1999) Storage and executive processes in the frontal lobes. Science 283:1657-1661.

Soto D, Humphreys GW, Rotshtein P (2007) Dissociating the neural mechanisms of memory-based guidance of visual selection. Proc Natl Acad Sci U S A 104:17186-17191.

Valenstein E, Bowers D, Verfaellie M, Heilman KM, Day A, Watson RT (1987) Retrosplenial amnesia. Brain 110:1631-1646.

Wagner AD, Shannon BJ, Kahn I, Buckner RL (2005) Parietal lobe contributions to episodic memory retrieval. Trends Cogn Sci 9:445-453.

Yantis S, Serences JT (2003) Cortical mechanisms of space-based and object-based attentional control. Curr Opin Neurobiol 13:187-193. 